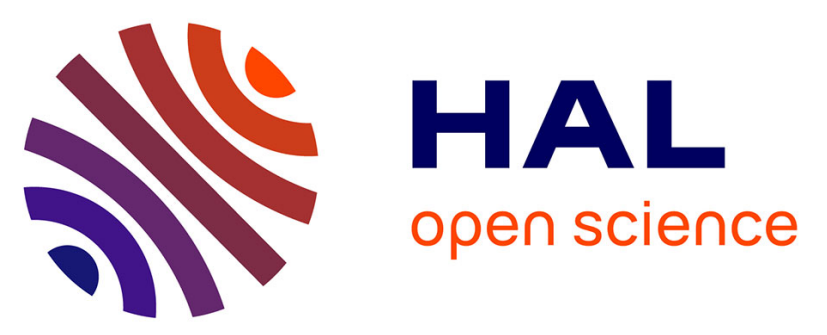

\title{
On the Use of Hackathons to Enhance Collaboration in Large Collaborative Projects - A Preliminary Case Study of the MegaM@Rt2 EU Project
}

Andrey Sadovykh, Dragos Truscan, Pierluigi Pierini, Gunnar Widforss, Adnan Ashraf, Hugo Bruneliere, Pavel Smrz, Alessandra Bagnato, Wasif Afzal, Alexandra Espinosa Hortelano

\section{To cite this version:}

Andrey Sadovykh, Dragos Truscan, Pierluigi Pierini, Gunnar Widforss, Adnan Ashraf, et al.. On the Use of Hackathons to Enhance Collaboration in Large Collaborative Projects - A Preliminary Case Study of the MegaM@Rt2 EU Project. Design, Automation And Test in Europe (DATE 2019), European Design and Automation Association (EDAA), Mar 2019, Florence, Italy. 10.23919/DATE.2019.8715247 . hal-02103168

\section{HAL Id: hal-02103168 https://hal.science/hal-02103168}

Submitted on 18 Apr 2019

HAL is a multi-disciplinary open access archive for the deposit and dissemination of scientific research documents, whether they are published or not. The documents may come from teaching and research institutions in France or abroad, or from public or private research centers.
L'archive ouverte pluridisciplinaire HAL, est destinée au dépôt et à la diffusion de documents scientifiques de niveau recherche, publiés ou non, émanant des établissements d'enseignement et de recherche français ou étrangers, des laboratoires publics ou privés. 


\title{
On the Use of Hackathons to Enhance Collaboration in Large Collaborative Projects
}

\author{
- A Preliminary Case Study of the MegaM@Rt2 EU Project -
}

\author{
Andrey Sadovykh \\ Innopolis University \\ Innopolis, Respublika Tatarstan, Russia \\ a.sadovykh@innopolis.ru \\ Dragos Truscan \\ Åbo Akademi University \\ Turku, Finland \\ dragos.truscan@abo.fi
}

\author{
Pierluigi Pierini \\ Intecs Solutions S.p.A. \\ Pisa, Italy \\ pierluigi.pierini@intecs.it
}

Gunnar Widforss
Mälardalen University
Västerås, Sweden
gunnar.widforss@ mdh.se

Hugo Bruneliere

IMT Atlantique, LS2N (CNRS) \& ARMINES

Nantes, France

hugo.bruneliere@imt-atlantique.fr

\author{
Pavel Smrz \\ Brno University of Technology \\ Brno, Czech Republic \\ smrz@ fit.vutbr.cz
}

\author{
Adnan Ashraf \\ Åbo Akademi University \\ Turku, Finland \\ adnan.ashraf@abo.fi
}

\author{
Wasif Afzal \\ Mälardalen University \\ Västerås, Sweden \\ wasif.afzal@mdh.se
}

\author{
Alessandra Bagnato \\ alessandra.bagnato@softeam.fr
}

\author{
Alexandra Espinosa Hortelano \\ Mälardalen University \\ Västerås, Sweden \\ alexandra.espinosa@mdh.se
}

\begin{abstract}
In this paper, we present the MegaM@Rt2 ECSEL project and discuss in details our approach for fostering collaboration in this project. We choose to use an internal hackathon approach that focuses on technical collaboration between case study owners and tool/method providers. The novelty of the approach is that we organize the technical workshop at our regular project progress meetings as a challenge-based contest involving all partners in the project. Case study partners submit their challenges related to the project goals and their use cases in advance. These challenges are concise enough to be experimented within approximately 4 hours. Teams are then formed to address those challenges. The teams include tool/method providers, case study owners and researchers/developers from other consortium members. On the hackathon day, partners work together to come with results addressing the challenges that are both interesting to encourage collaboration and convincing to continue further deeper investigations. Obtained results demonstrate that the hackathon approach stimulated knowledge exchanges among project partners and triggered new collaborations, notably between tool providers and use case owners.
\end{abstract}

Index Terms-Hackathon, Collaboration, Project

\section{INTRODUCTION}

MegaM@ $\mathrm{Rt}^{2}$ is a three-years project, funded by European Components and Systems for European Leadership Joint Undertaking (ECSEL JU) under the H2020 European program, that started in April 2017 [1], [2].

One of the main issues arose during the first plenary meeting, held in Rome, was the limited concrete and effective technical results in terms of cooperation between partners and experiments as well as the lack of progress related to the application of the available tools to the use cases. Therefore, we approached the following plenaries with a more balanced distribution of managerial and technical staff across meeting days, devoting a hackathon-like day as a strategy to foster collaboration between case study owners and tool/method providers on challenging technical issues.

We considered that the plenary meetings are the recommended venue for such events since at least one member of each project organization is typically present and available for face-to-face meetings. The latter are considered by different practitioners more efficient compared to virtual meetings [3].

The novelty of our approach is that we organize an internal technical workshop as a challenge contest. Case study partners submit in advance their challenges related to the project goals. These challenges should be concise to be experimented within 4 hours. The teams are then formed around those challenges. On the hackathon day, partners have 4 working hours to come with a result that should be both interesting and convincing.

In the following sections, we provide a high-level overview of the MegaM@ $\mathrm{Rt}^{2}$ project, challenges to manage technical collaboration in such a huge project, our approach for internal hackathons and preliminary results. We believe that the presented approach can be successfully applied to many other collaborative projects.

\section{OVERVIEW OF THE MEGAM@RT2 PROJECT}

The main goal of the MegaM@ $\mathrm{Rt}^{2}$ project is to create an integrated framework incorporating methods and tools for continuous system engineering and runtime validation and verification $(\mathrm{V} \& \mathrm{~V})$. The underlying objective is to develop and apply scalable model-based methods and tools, in order 
to provide improved productivity, quality and predictability of large and complex industrial systems.

One of the main challenges is to cover the needs coming from diverse and heterogeneous industrial domains, going from transportation and telecommunications to logistics. Among the partners providing case studies in the project, we can cite Thales, Volvo Construction Equipment, Bombardier Transportation or Nokia. These organizations have different product management and engineering practices, as well as regulations, commercial and legal constraints.

This results in a large and complex catalogue of requirements to be realized by the architecture building blocks at different levels of abstraction. Thus, the development of the MegaM@ $\mathrm{Rt}^{2}$ framework is based on a feature-intensive architecture and on a related implementation roadmap. The MegaM@ $\mathrm{Rt}^{2}$ framework plans to integrate 28 tools implementing the above-mentioned methods and satisfying requirements coming from 9 industrial case studies.

\section{ISSUES OF HUGE COLLABORATIVE PROJECTS}

Some instruments for collaborative projects gather very large and complex projects, sometimes involving more than 100 person years and sometime over 100 partners (beneficiaries). The average number of participants per project in Horizon 2020 in general is 4.69, in the second pillar alone the average is 5.91, in ICT it is 7.4, but in ECSEL it is 34.22 [16]. At the web page of ECSEL JU are 40 projects listed ranging from 9 to 109 participants [4].

There is an assumption that the larger a project is, the more complementary the resources of different partners can be, and the larger the impact as well, but large consortia may not be that efficient. Thus, large projects are not good per se.

To some degree, a project thrives with more partners of different kinds. Different views, backgrounds, competencies, networks, focuses etc., make the collaboration better and enrich the work because of complementary and mutual learning.

However, there are some evidences that over a certain size, the larger a project is, the lower the effect of complementary becomes. This is because of larger uncertainties, higher transaction costs and a cognitive distance among partners [4] [5]. Cognitive distance poses both a problem and an opportunity for collaboration, in that a large distance provides the potential for novelty and creativity (i.e. to learn something new) but at the same time makes understanding more difficult between the parties involved [4]. The dimension of the projects poses several challenges for its management. Hence, it is not likely that all the staff from two partners ever meet in the project, as it was concluded for another similar project [6] [7] [5].

Thus, in large, collaborative multi-partner projects there is a distance between the partners, that has to be addressed and bridged. The concept of distance involve different aspects:

- the kinds of partner organizations that span from academia to small, medium and large enterprise;

- the kinds of project members expertise and seniority, varying between industrial employees or academic pro- fessors, younger researchers and developers, managers, administrators, entrepreneurs etc.;

- the difference in national cluster either in terms of number of organizations and, most importantly, in funding and budget restrictions due to different national rules in economic support;

- last, the cultural heritage that affects the personal approach to the job activities and relationships.

We experienced a need to bridge this distance, since during the kick-off and the first plenary meeting, held in Rome, we received substantial feedback from the staff complaining that the content was too administrative or managerial. Instead of just using the plenary meetings to planning and reporting, we thought we should take advantage of our differences and plan the meeting in a manner that challenges also the technical competence represented by the most of the partners.

\section{A. Challenges}

In the following, we enumerate several challenges that can be found in European projects such as MegaM@ $\mathrm{Rt}^{2}$.

Dimension and partner organization. In the case of MegaM@ $\mathrm{Rt}^{2}$, we have 27 beneficiaries, distributed among academia (7 universities and 3 research centers), Small and Medium Enterprises (8 SMEs) and Large Enterprises (9 LEs) from 6 countries (Finland, Sweden, Czech Republic, Italy, Spain and France), slightly below the average ECSEL project. Anyhow, we have well over 120 participants/members directly involved in the project.

National clusters. The differences are partially related by the different composition of researches, SMEs and LEs. But the most significant issue is related to the different funding rates. The European Commission (EC) covers $25 \%$ to $35 \%$ of the total budget. National support vary significantly based on country and type of organization. National funding rate for LEs is null in France, as small as $10 \%$ in Italy, increasing up to $25 \%$ in Finland. SMEs span from $15 \%$ to $35 \%$. Academia and research centers may receive up to $60 \%$ of total budget. These facts may impact the planning and the level of participants expertise engaged by each organization in the project.

Cultural heritage. Studies about differences in social relationships and habits represent a huge discipline. Hofstede Insights apply a structural approach and model to understand and exploit cultural knowledge for business. A comparison chart [8] classifies the differences between European countries into six categories (see for instance "Fig. 1"):

- Power Distance: The extent to which the less powerful members of society accept that power is distributed unequally;

- Individualism: (Individualist versus Collectivist) Collectivism: people belong to in-groups (families, organizations, etc.) who look after them in exchange for loyalty. Individualism: people only look after themselves and their immediate family;

- Masculinity: The dominant values in society are achievement and success versus caring for others and quality of life; 
- Uncertainty/avoidance: The extent to which people feel threatened by uncertainty and ambiguity and try to avoid such situations.

- Long term orientation: The extent to which people show a pragmatic or future-oriented perspective rather than a normative or short-term point of view

- Indulgence: The extent to which people try to control their desires and impulses.

Expertise and seniority. Last but not least, we can classify the participants from the same organization as business managers and technical persons. The former participate mainly in coordination activities, while the latter are the ones who develop and deliver the actual results, so they have to be involved to interact with counterparts from other organizations.

\section{B. Common drawbacks of traditional plenary meetings}

The authors have participated in more than 30 collaborative research projects. Collectively we can identify several common issues with traditional approaches for project regular plenary meetings. We received many complaints that the plenary meetings are very administrative. Usually, a plenary is divided in slots for presentation by various partners (e.g. Work Package leaders) about the current state of the project and future plans. Many times, the discussions are kept at minimum. Many participants feel disengaged and consider plenary meetings as a waste of time. As consequence, many partners apply cost savings and send managers only without involving the technical staff, while, in fact the technical persons is actually producing the deliverables and would stronger benefit from tighter links with colleagues in other organizations working on the same deliverables. Thus, the output of plenary meetings becomes questionable.

We decided to re-think the plenary organization focusing the multiple objectives of the meeting: the administrative ones, to address the housekeeping issues related to the project status and its evolution, but the technical ones as well to trace the guidelines and focus the objectives of the new developments. To comply with this last point, we have directed towards a revision of the hackathon concept in order to effectively apply it to our needs.

\section{HACKATHON RELATED WORK}

In recent years, hackathons have emerged as a popular way of attracting people with technical backgrounds to work together in teams and quickly produce working solutions. Nandi and Mandernach [9] investigated informal learning aspects of hackathons and reported that hackathons provide a structure for increased informal learning, promote peer learning and boost motivation of the participants to learn new skills.

Anslow et al. [10] proposed to augment computer science curriculum with datathons (hackathons focusing on data analytics) and presented their results from four datathons involving computer science students and volunteer data scientists. In these datathons, the teams explored different datasets and helped the participating organizations find better uses of their data.

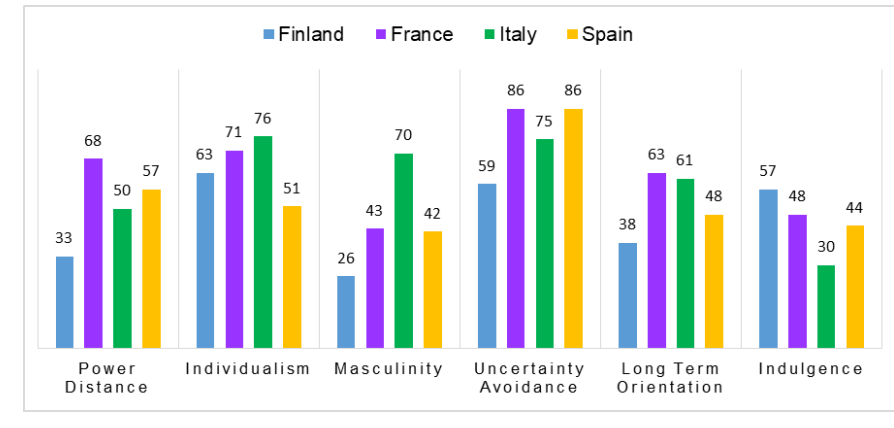

Fig. 1. Hofstede country charts comparison example [8]

Decker et al. [11] argued that hackathon culture is usually intimidating. They proposed an alternative non-competitive, community-based format for hackathons called Think Global Hack Local (TGHL), which is aimed at making hackathons more inclusive and fun for all participants. They also reported results from two successful TGHL events involving non-profit organizations and computer science students.

Hackathons are not limited only to education and learning environments. They are also useful for rapidly exploring new business ideas and producing software prototypes [12]. However to generate revenue and create real business value, promising prototypes must be developed further and transformed into finalized products.

Raatikainen et al. [13] presented their experience from a hackathon organized to assess and validate the requirements and design of a device-centric cloud ecosystem. They reported that hackathon is a useful approach for this kind of task as it allows an efficient assessment of software artifacts and provides directions for further development and improvement of such systems. The hackathon resulted in a set of new requirements and three prototypes. Raatikainen et al. [13] also highlighted some social benefits of hackathons including collaboration, inspiration, and motivation.

Komssi et al. [12] reported results from five hackathon events, each having a different purpose. The most relevant of these hackathons was aimed at a collaboration among companies and research organizations. The hackathon resulted in six working prototypes and continued collaboration between two companies to further develop the ideas and prototypes.

Rosell et al. [14] introduced the concept of internal hackathons, which are organized particularly for the internal employees of an organization. They also described some benefits of internal hackathons and reported their experience from a successful internal hackathon which was organized to find new creative ways of using a new communication protocol. The internal hackathon extended the usual hackathons in two ways. First, a special attention was given to the preparation of the participants. Secondly, the hackathon was open to employees in both technical and non-technical positions. Out of 120 participants, $48 \%$ came from non-development departments while $13 \%$ had a purely non-technical background. The hackathon teams produced a wide range of solutions and identified six 
areas of innovation and improvement for the communication protocol.

Frey and Luks [15] presented innovation-driven hackathons as a mean for overcoming organizational obstacles and challenges which hinder and slow down innovation within medium and large sized companies. They suggested to invite best people with different skills and backgrounds from both within and outside the organization to participate in compact 1-3 days events. They also proposed that the main event should have time-boxed iterations comprising four phases: problem definition, solution alternatives, prototypes, pitch and feedback. The final pitch should be given in front of a jury, which may select one or more concepts or prototypes for further development in the context of the project.

\section{THE HACKATHON APPROACH IN MEGAM@RT2}

The main goal of the approach presented in this paper is to boost the project development by increasing the efficiency of the plenary meetings:

- involving at plenary meetings not only management personnel responsible for project coordination, but also actual doers (i.e. technical people) in order to foster collaboration and cross-fertilization among partners;

- creating positive synergies between use case and technology providers;

- helping use case providers to bootstrap the baseline experiments by providing use case providers dedicated technology demonstrators on a clearly limited subset of their specific industrial challenge (based on realistic concrete material, e.g. models, code, etc.);

- using the demonstrators to track project progress with respect to project deliverables, illustrating results as well as spotting the innovation at early stages;

- ensuring that all the case studies have several partners involved.

In order to address the above goals, we devised an internal hackathon event that we applied at two plenary meetings and which is planned to be also organized in future plenaries. Based on the above arguments, we decided to design a custom internal hackathon that will enhance the collaboration and exchange of ideas between different project partners. Our approach to internal hackathons is described in the following.

\section{A. Specifics of our internal hackathon.}

In the MegaM@ $\mathrm{Rt}^{2}$ project we apply a customized version of the hackathon, involving 5 prerequisites:

- Technical staff must be involved;

- For each challenge proposed by a use case owner, there should be at least one technology provider subscribed;

- Defined time boxes for the work;

- Competition, entertainment and small prizes;

- Inclusive environment where everybody feels concerned.

Our hackathon process comprises of three main phases: before, during, and after, each with own set of activities, as follows.
Before phase is characterized by a call for hackathon: case study providers are required to prepare hackathon challenges (i.e. a well-defined and limited experiment related to use cases that can be explored in a half day work) and announce them to the rest of the participants. Tool and technology providers subscribe to these hackathon challenges proposing methods and tools that can solve the challenge. This approach allows all the involved participants to identify punctual challenges and prepare in advance for them, by providing the corresponding documentation, artifacts and tools, and by choosing the corresponding technical personnel to participate in the event.

During the hackathon session, each hackathon challenge is presented by the case study providers as a technical pitch in the morning of the event. On one hand, this allows the entire audience to be aware of the details of the challenge. On the other hand, it gives the possibility for additional participants (i.e. not already subscribed) to join the hackathon challenge. Then working groups are formed for each challenge to work in parallel in 2 sessions of 4 hours each.

After the hackathon sessions, each challenge provider gives in plenum a short overview of the main outcomes of the work and plans for future collaboration. In addition, all plenary participants are asked to evaluate the results of each challenge using an anonymous online voting system.

\section{B. Evaluation metrics}

Four aspects are evaluated: Technical innovation - how novel is the presented result, is it a breakthrough or an evolution?; Exploitation potential - can this demo be a step to generate revenues, foster market access to tool providers and help case study providers to improve their own developments?;

Technological readiness - (for demonstration purposes) - does the team work look as a finished demonstration that we can reuse?; and Entertainment (fun) - does the team present the results in a way that is both instructive and easy to digest? An example is given in "Fig. 2".

The best demos/presentations voted by the audience are selected as showcases for different project dissemination activities.

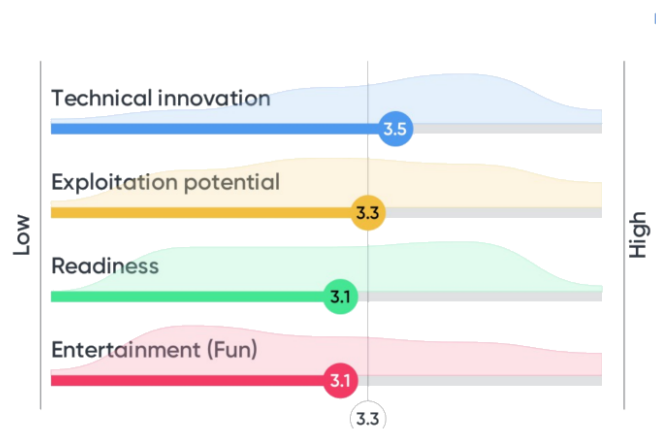

Fig. 2. Example of challenge evaluation

Additional questions helped to understand the acceptance and the adequacy of the plenary tuning among technical and 
What was the best thing with the plenary?

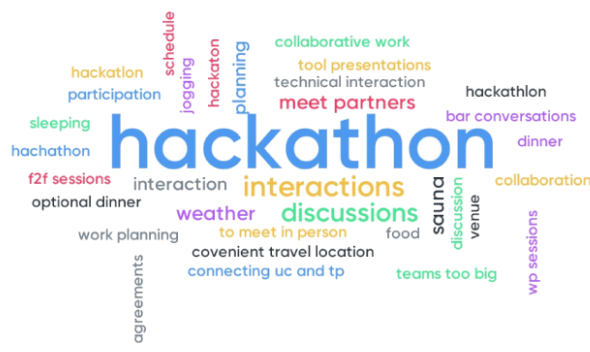

43

Fig. 3. Best part of the plenary meeting based on participants' votes

managerial sections and the general feeling on hackathon experience. For instance, when we asked the plenary participants to specify the three best parts of the plenary the hackathon received the most votes, as shown in "Fig. 3".

Furthermore, the participants provided positive feedback when asked to comment on the hackathon experience as illustrated in "Fig. 4".

Last but not least, the vast majority of the participants considered the progress generated by the internal hackathon as significant. They were planning to work further on the ideas developed during the hackathon after the plenary meeting in order to foster inner project collaboration and boost the project overall progress.

Finally, they voted to continue with such an approach at the following plenary meetings.

\section{CONCLUSIONS AND LESSON LEARNED}

In this paper, we presented an internal hackathon approach that we defined and applied in the context of the MegaM@ $\mathrm{Rt}^{2}$ project in order to boost project collaboration and development. After the first project's plenary meeting held in Rome that highlighted the lack of effective technical results, the internal hackathon initiative has been applied in the two following plenary meetings in Helsinki and Paris. Based on the positive feedback from the project participants, we plan to apply it again in future ones.

The best hackathon results of each plenary meeting have been selected for dissemination activities. In addition, they were presented in the first official review meeting of the project, where both the approach and the results received the appreciation of the project reviewers.

The long-term effects are still under observation and need to be quantified in a more formal way, but some significant benefits are already tangible and concrete. So far, we have observed the following immediate effects:

- General improvement of the project working atmosphere;

- Continuation of the hackathon work on new research lines, and new applications of project tools to use cases;

- Easier development progress status tracking of use cases and tools, comprehension of practical demonstration;

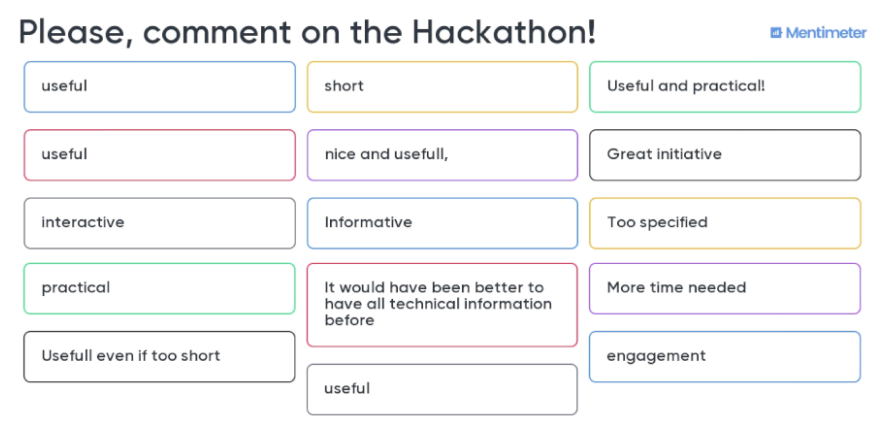

33

- Significant improvement on partner interactions either among use cases and tools providers and between tool providers, that also identified new opportunities to integrate with each others tools (sometime complementary) or new features to improve their offers.

However, we will continue to improve and evaluate our internal hackathon approach in the next plenary meetings while keeping in mind the following risks:

- Hackathons produce prototypes used as proof-ofconcepts, that should not be considered as final products;

- Hackathons are focused on well-delimited challenges. The longer-term focus can be missed without proper follow-up and monitoring of the related activities;

- Hackathons cannot be used as a day-to-day practice, since the daily effort is very intense and the team may easily burn out.

The main mitigation for the above risks is to repeatedly make the project partners aware of them both at the plenary meetings and throughout the project. In addition, we are considering to adjust the hackathon sessions over several days of the plenaries, and interleaving them with the project coordination sessions to make the two technical and administrative aspects more cohesive.

\section{ACKNOWLEDGMENT}

This work has received funding from the Electronic Component Systems for European Leadership Joint Undertaking under grant agreement No 737494. This Joint Undertaking receives support from the European Unions Horizon 2020 research and innovation programme and Sweden, France, Spain, Italy, Finland, the Czech Republic.

\section{REFERENCES}

[1] W. Afzal et al., The MegaM@ $\mathrm{Rt}^{2}$ ECSEL project: MegaModelling at Runtime Scalable model-based framework for continuous development and runtime validation of complex systems, Microprocessors and Microsystems, vol. 61, pp. 8695, 2018 [Online]. Available: http://dx.doi.org/10.1016/j.micpro.2018.05.010

[2] MegaM@Rt ${ }^{2}$ Project Website. [Online]. Available: https://megamart2ecsel.eu/. [Accessed: 02-Oct-2018] 
[3] N. Morgan, 5 Fatal Flaws with Virtual Meetings, Forbes, 02-Oct-2012 [Online]. Available: https://www.forbes.com/sites/nickmorgan/2012/10/02/5-fatal-flawswith-virtual-meetings/

[4] ECSEL Joint Undertaking Electronic Components and Systems for European Leadership, Projects. [Online]. Available: https://www.ecsel.eu/projects

[5] B. Nooteboom, Inter-firm Alliances: Analysis and Design. Psychology Press, 1999 [Online]. Available: https://books.google.com/books/about/Inter_firm_Alliances.html?hl=\&id=yP8IFFQm-oC

[6] Y. E. Spanos, Conditionally-mediated effects of scale in collaborative R\&D, J. Technol. Transf., vol. 37, no. 5, pp. 696714, 2011 [Online]. Available: http://dx.doi.org/10.1007/s10961-011-9218-7

[7] G. Widforss and M. Rosqvist, On top of the consortium: Keeping control of consortium-building in ICT R\&D programmes, in 2015 IEEE International Conference on Engineering, Technology and Innovation/ International Technology Management Conference (ICE/ITMC), 2015 [Online]. Available: http://dx.doi.org/10.1109/ice.2015.7438648

[8] Hofstede Insights - Compare Countries. [Online]. Available: https://www.hofstede-insights.com/product/compare-countries/. [Accessed: 11-Oct-2018]

[9] A. Nandi and M. Mandernach, Hackathons as an Informal Learning Platform, in Proceedings of the 47th ACM Technical Symposium on Computing Science Education - SIGCSE 16, 2016 [Online]. Available: http://dx.doi.org/10.1145/2839509.2844590

[10] Anslow, Craig and Brosz, John and Maurer, Frank and Boyes, Mike, Datathons: An Experience Report of Data Hackathons for Data Science Education, in Proceedings of the 47th ACM Technical Symposium on Computing Science Education, 2016, pp. 615620 [Online]. Available: http://doi.acm.org/10.1145/2839509.2844568

[11] A. Decker, K. Eiselt, and K. Voll, Understanding and improving the culture of hackathons: Think global hack local, in 2015 IEEE Frontiers in Education Conference (FIE), 2015 [Online]. Available: http://dx.doi.org/10.1109/fie.2015.7344211

[12] Marko Komssi, Danielle Pichlis, Mikko Raatikainen, Klas Kindstrm, Janne Jrvinen, What are Hackathons for?, IEEE Software, vol. 32, no. 5, pp. 6067, 2015 [Online]. Available: http://dx.doi.org/10.1109/MS.2014.78

[13] M. Raatikainen, M. Komssi, V. D. Bianco, K. Kindstom, and J. Jarvinen, Industrial Experiences of Organizing a Hackathon to Assess a Device-centric Cloud Ecosystem, in 2013 IEEE 37th Annual Computer Software and Applications Conference, 2013 [Online]. Available: http://dx.doi.org/10.1109/compsac.2013.130

[14] B. Rosell, S. Kumar, and J. Shepherd, Unleashing innovation through internal hackathons, in 2014 IEEE Innovations in Technology Conference, 2014 [Online]. Available: http://dx.doi.org/10.1109/innotek.2014.6877369

[15] F. J. Frey and M. Luks, The innovation-driven hackathon, in Proceedings of the 21st European Conference on Pattern Languages of Programs - EuroPlop 16, 2016 [Online]. Available: http://dx.doi.org/10.1145/3011784.3011794

[16] H2020 participation - full details, 2018-10-09 https://webgate.ec.europa.eu/dashboard/sense/app/ 